\title{
An EIRP Measurement Method for Base-Station Antennas Using Field Strengths Measured along a Single Straight Line
}

\author{
Soon-Soo Oh and Young-Hwan Lee \\ Radio Technology Research Department, ETRI, 218 Gajeongno, Yuseong-gu, Daejeon 305-700, Republic of Korea \\ Correspondence should be addressed to Soon-Soo Oh; ssoh@etri.re.kr
}

Received 9 November 2012; Revised 28 February 2013; Accepted 15 March 2013

Academic Editor: Michael Yan Wah Chia

Copyright ( 12013 S.-S. Oh and Y.-H. Lee. This is an open access article distributed under the Creative Commons Attribution License, which permits unrestricted use, distribution, and reproduction in any medium, provided the original work is properly cited.

\begin{abstract}
We describe an EIRP measurement technique for a base-station antenna. The proposed method especially can be applied to the base-station antenna installed in real environments. Fresnel region measurement method is an optimal technique to avoid the farfield multipath interference, and, furthermore, it could shorten the measurement time. For detecting only the field strengths along a single straight line, we also propose a simple phase-retrieval method. For verification, a simulation and experiment have been performed. An anechoic chamber was utilized in this paper before the real environment test with the outdoor measurement system. The transformed far-field pattern and EIRP agree closely with the reference data within a valid angle. The proposed method can be applied for the EIRP in situ measurements without moving a vehicle loading the EIRP measurement apparatus.
\end{abstract}

\section{Introduction}

As wireless communication services with low power are further developed, protection from high-power radiation devices and facilities such as base-stations and digital broadcasting stations is becoming more important [1-5]. The equivalent isotropically radiated power (EIRP) is generally used to restrict the amount of radiation power from wireless devices, which is defined as EIRP $=G_{t} \times P_{t}$, where $G_{t}$ is the gain of the transmitting antenna, and $P_{t}$ is the input power to the transmitting antenna [6-8]. Generally, EIRP measurement is performed before the installation of the base station. However, the communication service provider could violate the upper limit of EIRP, and the inspection after the installation of the base station is required [9].

The EIRP measurement of a base-station antenna installed in real environments is a major challenge. First, base-station antenna transmissions invariably suffer from multipath interference when they are measured in the farfield region due to reflections from buildings, the ground, trees, and so forth. The near-field method seems to be a good alternative, but probes placed close to a base-station antenna may disturb communications. Furthermore, planar or cylindrical scanning is required, which results in long scanning and processing times [10-13]. For these reasons, the Fresnel field measurement method [14-17] can be an optimal technique. Second, only magnitude data can be detected, as the base station is in operation and the reference phase cannot be obtained. The phase-retrieval techniques based on the data obtained on the two planar or cylindrical planes have been developed [11-13], but it is difficult to detect the data on two planes in real situations due to the presence of physical obstacles or other base stations [16]. Therefore, the scanning method without moving a vehicle loading the EIRP measurement apparatus is required.

In this paper, Fresnel region measurement and a simple phase-retrieval method have been proposed in order to obtain the EIRP of a base-station antenna in a real situation. Only scanning along a single line is required. For verification, the simulated and experimental results of tests performed in an anechoic chamber are described and discussed.

\section{Theory}

The scheme of proposed method is illustrated in Figure 1. The base-station antenna is fixed and the probe is moved with separation distance $R_{1}$. 


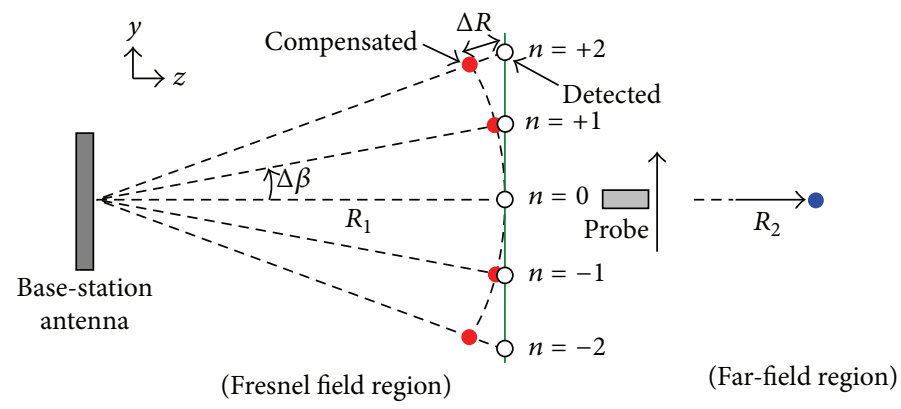

FIGURE 1: Scheme of the proposed method.

As shown in Figure 1, the movement trace of the probe is linear. Since the base-station antenna is elongated, the near-field measurement method requires cylindrical scanning. Therefore, Fresnel field measurement method [1417] is faster than the near-field measurement method. The detected magnitudes are compensated by $\left|E_{R_{1}, \text { compensated }}\right|=$ $\left|E_{R_{1} \text {, detected }}\right| *\left(1+\Delta R / R_{1}\right)$, and this approximation has been proved to be accurate in [15]. In Figure 1, the white circles indicate the detection points while the corresponding compensated fields are marked in red. The differential angle $\Delta \beta$ is the angle between the detection points, which is determined by $\Delta \beta=\lambda / L_{y}[15]$, where $\lambda$ is the wavelength, and $L_{y}$ is the length in the $y$-direction of base-station antenna. The number of required data $N$ for one far-field direction is $N=$ $L_{y}^{2} / \lambda R_{1}+1$ for moderate accuracy and $N=L_{y}^{2} / \lambda R_{1}+5$ for high accuracy [16]. Figure 1 shows the case of $N=5$.

As mentioned in Section 1, the detected fields contain only the magnitude, and therefore the phase information must be retrieved. In this paper, a fast and simple phaseretrieval technique is proposed as follows.

(I) Estimate the complex value of the distribution $f(x, y)$ over the artificial aperture. The distribution is based only on the following simple formula:

$$
f(x, y)=\cos ^{p_{1}}\left(c_{1} y\right)+j \sin ^{p_{2}}\left(c_{2} y\right),
$$

where $p_{1}, p_{2}, c_{1}$, and $c_{2}$ are the tuning parameters. The base-station antenna is elongated, and thus the distribution along the $x$-direction is uniform, and only the variation along the $y$-direction is required. The aperture is subdivided by $\lambda / 8$ spacing, which has been verified to provide accurate results based on our simulation and experiment.

(II) Calculate the field at $R_{1}$ using

$E_{R_{1}}=\iint_{S} f(x, y) e^{+j k(x \sin \alpha+y \cos \alpha \sin \beta)} e^{-j\left(\pi / \lambda R_{1}\right)\left(x^{2}+y^{2}\right)} d x d y$,

where $\alpha$ is the angle from the $y-z$ plane and $\beta$ is the angle from the $x-z$ plane.

(III) Compare the calculated magnitude pattern $\left|E_{\text {cal }}\right|$ from (2) and the detected pattern $\left|E_{\text {meas }}\right|$ in the

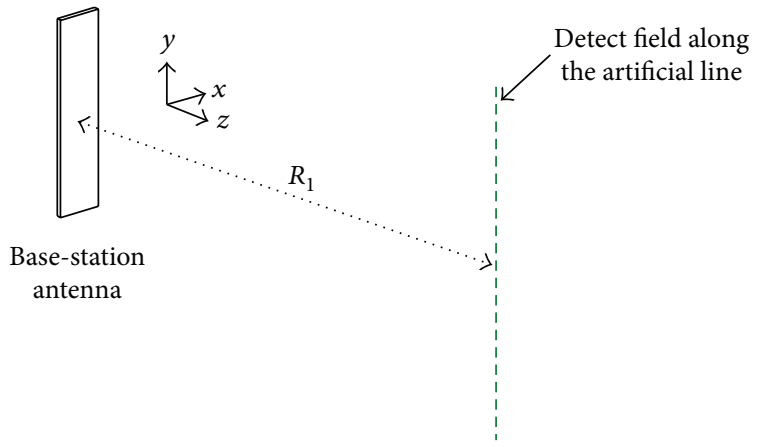

FIGURE 2: Simulation model of base-station antenna.

normalized value. The minimization factor is $\sum \|\left. E_{\text {cal }}|-| E_{\text {meas }}\right|^{2}$.

(IV) Determine the phase distribution at $R_{1}$ using (2), if the two patterns agree over the lower level range of about $15 \mathrm{~dB}$. If not, go back to procedure (I) and adjust parameter $p$, and then process procedures (II) and (III) until the two patterns agree well.

Since simple formulas (1) and (2) are utilized, the proposed phase-retrieved technique is fast. One might think that the formula (1) might be inappropriate for other antennas. Practically, however, the preliminary information for a basestation antenna, such as the beam pattern, can be easily obtained since they have already been submitted to the basestation certification agency. Thus, an equation such as (1) can be prechecked, and another simple equation can be built.

After estimating the phase, the measured magnitude and retrieved phase at $R_{1}$ are inserted into (3) [16] and transformed into the value at the far-field region, which results in $P_{R_{2}}$, as shown in Figure 1,

$$
E_{R_{2}}=\frac{R_{1}}{R_{2}} e^{-j k\left(R_{1}-R_{2}\right)} \sum_{n=-N}^{+N} k_{m n} E_{R_{1}}(\beta+n \Delta \beta),
$$

where $k_{m n}$ is the Fourier coefficient. Now, EIRP is calculated by the Friss transmission formula [10],

$$
\mathrm{EIRP}=\frac{P_{R_{2}}}{G_{\text {Probe }}} \times\left(\frac{4 \pi R_{2}}{\lambda}\right)^{2},
$$

where $G_{\text {probe }}$ is the gain of the receiving probe. 


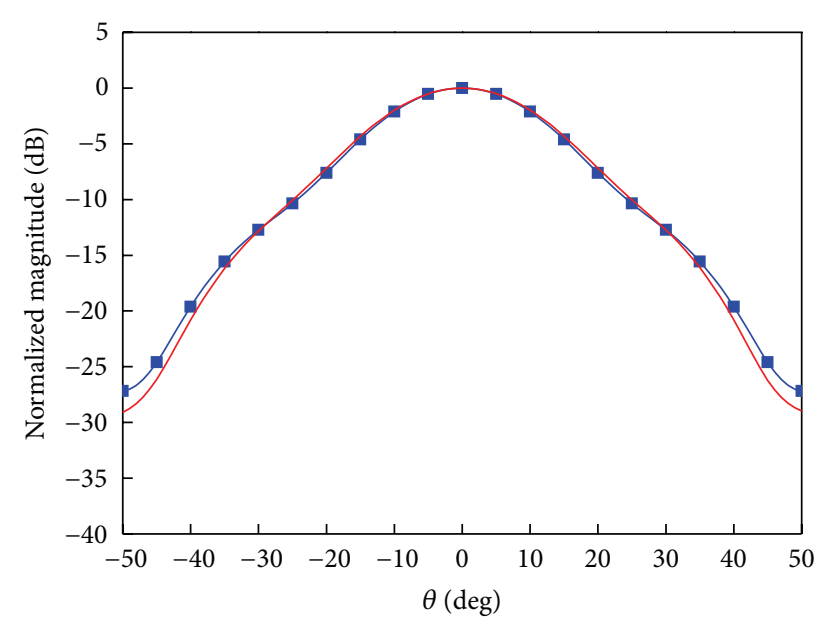

(Simulation)

- Detected

$\rightarrow$ Retrieved

(a)

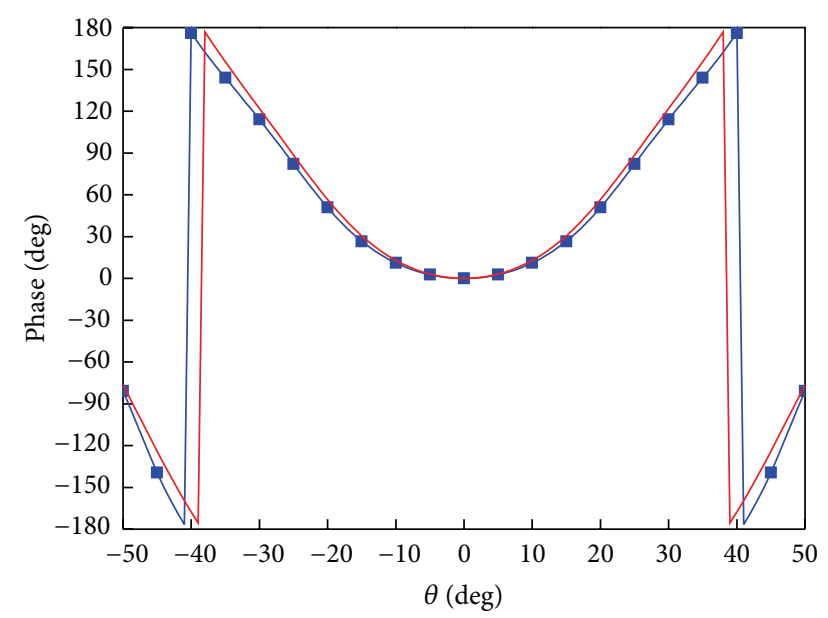

(Simulation)

- Detected

- Retrieved

(b)

Figure 3: Fresnel field pattern from the simulation: (a) detected and retrieved magnitudes and the (b) exact and retrieved phases.

\section{Simulated and Experimental Results}

For verification of the proposed method, a simulation was performed with the aid of commercial software, Ansoft's HFSS [18]. A base-station antenna for a Personal Communication Service (PCS) was chosen in the experiment for convenience. The simulation model is illustrated in Figure 2. The frequency was set to $1.8 \mathrm{GHz}$. A simple rectangular conducting plate with a dimension of $0.15 \mathrm{~m} \times 0.6 \mathrm{~m}$ in the $x$ - and $y$-directions, respectively, was excited by the wave-port model. Since the input power to the antenna was $0 \mathrm{~dB}$ and the simulated far-field gain was $G_{t}=15.44 \mathrm{dBi}$, the reference EIRP can be calculated as EIRP $=G_{t}+P_{t}=15.44+0.0=15.44 \mathrm{~dB}$.

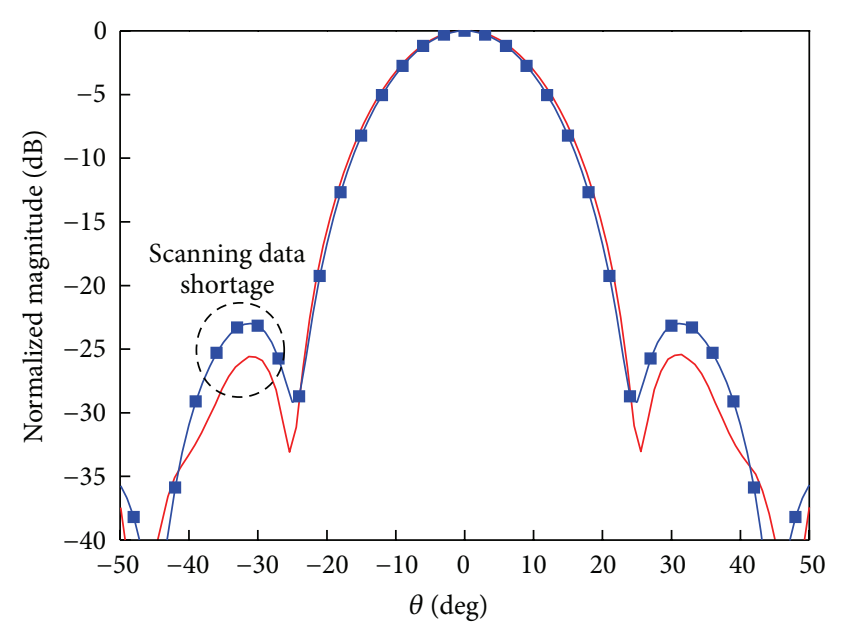

(Simulation)

- Transformed

$\rightarrow$ Reference

FIGURE 4: Transformed and reference far-field patterns from the simulation.

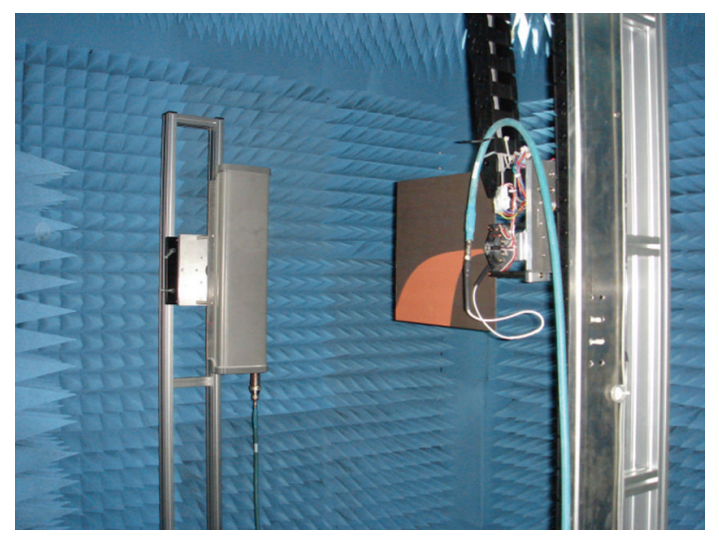

FIgURE 5: Photograph of experiment of the base-station antenna in a small anechoic chamber.

As shown in Figure 2, the radiated fields from the antenna were detected along a straight line with the length of $2.0 \mathrm{~m}$. The separation distance between the base-station antenna and probe is $R_{1}=0.6 \mathrm{~m}$, which is about $1 / 8$ of the minimum far-field distance. The phase-retrieval technique described in Section 2 was performed. After several iterations of pattern comparison, the final values were $p_{1}=1.1, c_{1}=1.0, p_{2}=0.0$, and $c_{2}=0.0$.

The detected- and retrieved-magnitude fields are plotted in Figure 3(a). The retrieved phase is plotted in Figure 3(b). For the comparison, the exact phase along the straight line is also plotted in Figure 3(b). They agreed very well.

The transformed far-field pattern, $P_{R_{2}}$, is shown in Figure 4. For comparison, the reference far-field pattern acquired from the far-field simulation is also shown in Figure 4 . The normalized patterns agree closely over the range of $\theta=-20^{\circ}$ to $+20^{\circ}$. The disagreement outside this range was due to the lack of scanning data [19]. The extrapolation 


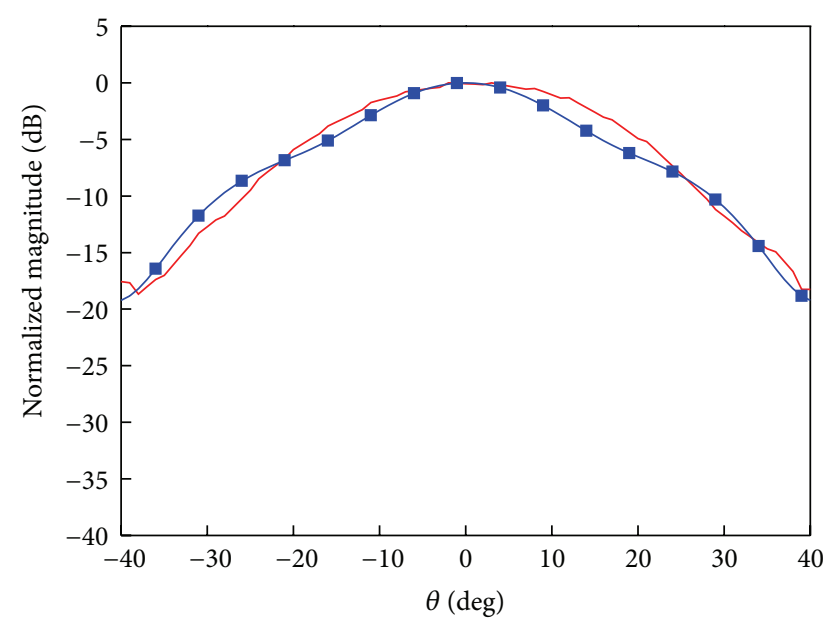

(Experiment)

- Detected

- Retrieved

(a)

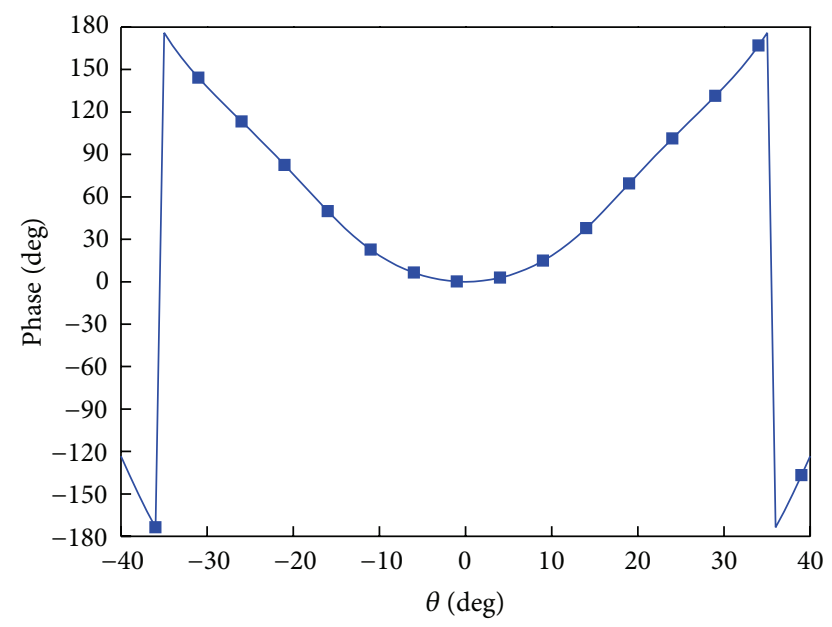

(Experiment)

$\rightarrow$ Retrieved

(b)

FIGURE 6: Fresnel field pattern from the experiment: (a) detected and retrieved magnitudes and the (b) retrieved phase.

technique expanding the scanned data outside the valid angle could improve the accuracy [19]. The $P_{R_{2}}$ at $\theta=0^{\circ}$ was $19.52 \mathrm{~dB}$ and thus EIRP $=15.18 \mathrm{~dB}$ from (4). The difference between the transformed and reference EIRPs was only $0.26 \mathrm{~dB}$

For the experimental verification, the base-station antenna is installed in a small anechoic chamber, as shown in Figure 5. The frequency was set to $1.8 \mathrm{GHz}$, and the antenna dimensions were $0.15 \mathrm{~m} \times 0.6 \mathrm{~m}$ in the $x$ - and $y$ directions, respectively. The far-field gain of the base-station antenna was measured in a medium-sized anechoic chamber satisfying the far-field criterion, and the resultant gain was $G_{t}=13.65 \mathrm{dBi}$. The input power to the base-station antenna

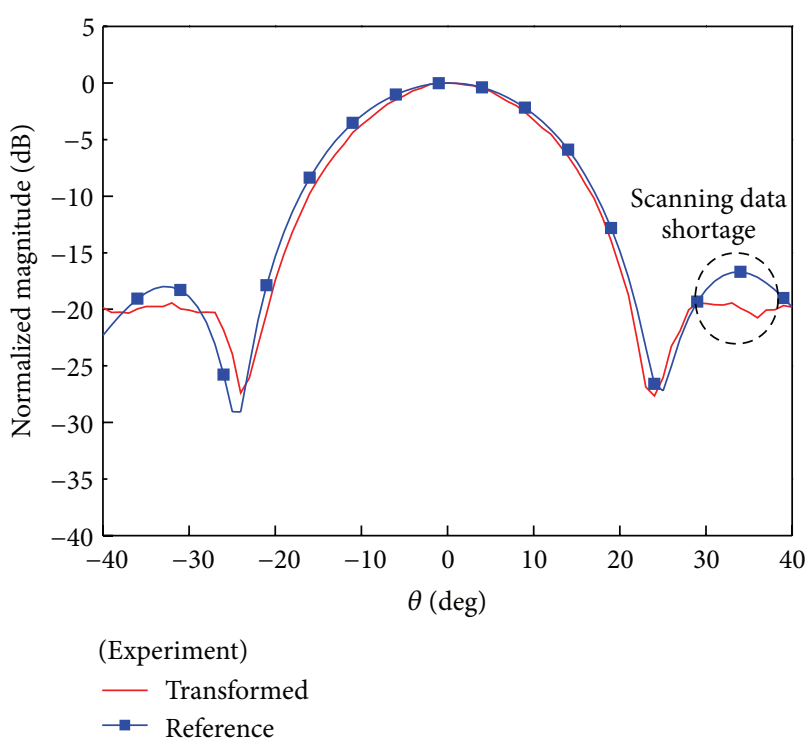

Figure 7: Transformed and reference far-field patterns from the experiment.

was $P_{t}=6.7 \mathrm{dBm}$. The exact EIRP from EIRP $=G_{t} \times P_{t}(\mathrm{~dB})$ was thus $20.36 \mathrm{dBm}$.

As shown in Figure 5, the field detecting probe was designed with a tapered slot antenna, and the gain was $G_{\text {probe }}$ $=9.21 \mathrm{dBi}$. Since this experimental verification was performed in the in-door chamber, the bulky probe is appropriate. However, if one performs in the outdoor environment, a miniature probe would be more appropriate in strong wind conditions.

The separation distance between the base-station antenna and probe is $R_{1}=0.6 \mathrm{~m}$. Meanwhile, the far-field distance of the utilized antenna is larger than $4.7 \mathrm{~m}$. The magnitude field is detected and then compensated as shown in Figure 6(a). Now, the phase-retrieval technique described in Section 2 was performed. After several iterations for parameters in (1), the final values were $p_{1}=1.0, c_{1}=0.8, p_{2}=0.0$, and $c_{2}=$ 0.0 . The retrieved magnitude is compared to the detected magnitude as shown in Figure 6(a). They almost agreed.

The retrieved phase is also plotted in Figure 6(b). Since the data-acquisition software could detect only the magnitude, the retrieved phase curve as shown in Figure 6(b) cannot be compared with the exact one, but the agreement of simulation results as shown in Figure 3(b) can guarantee the accuracy of the retrieved phase curve.

The detected magnitude and retrieved phase were used for the transformation from the Fresnel field at $R_{1}=0.6 \mathrm{~m}$ to the far field at $R_{2}=4.7 \mathrm{~m}$. The transformed far field pattern, $P_{R_{2}}$, is shown in Figure 7. For comparison, the farfield pattern acquired in the medium-sized anechoic chamber is also shown in Figure 7. The normalized patterns agreed closely over the range of $\theta=-20^{\circ}$ to $+20^{\circ}$. The disagreement outside this range was due to the lack of scanning data caused by the scanning limitations in the $y$-direction. The transformed $P_{R_{2}}$ at $\theta=0^{\circ}$ was $-22.12 \mathrm{~dB}$, and thus EIRP $=$ $19.66 \mathrm{dBm}$ from (4). The difference between the transformed 


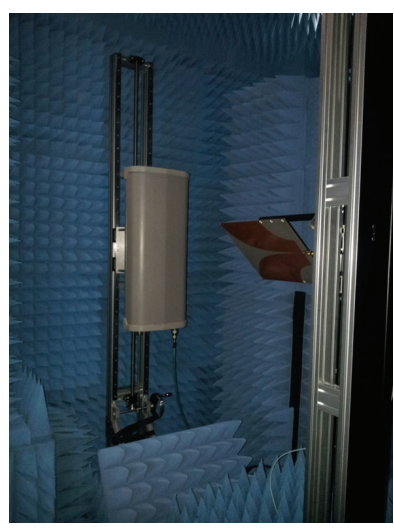

(a)

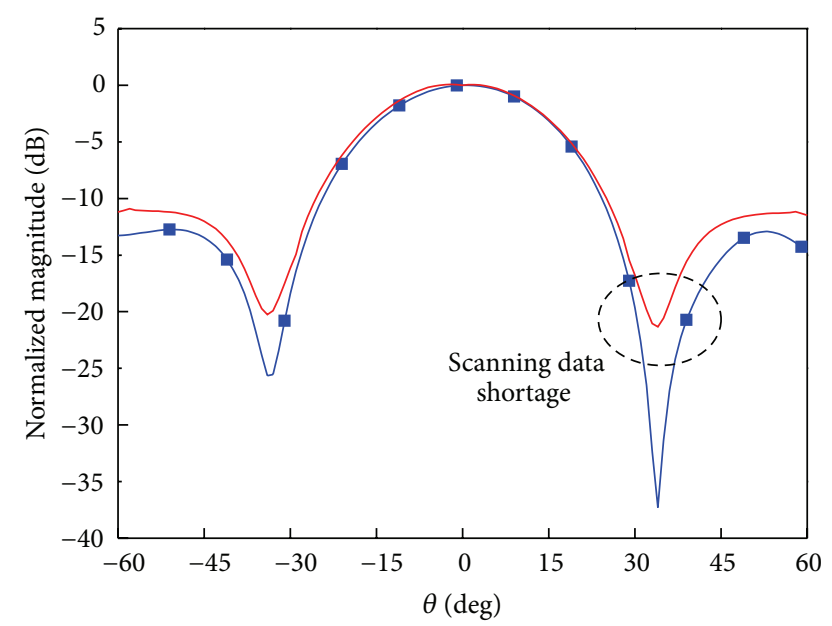

(Experiment)

- Transformed

$\rightarrow$ Reference

(b)

FigURE 8: Another experiment of the base-station antenna operating at $859 \mathrm{MHz}$ (a) Photograph (b) Transformed and reference far-field pattern.

and reference EIRPs was $0.69 \mathrm{~dB}$. This is a slightly large error. The error from the simulation was only $0.26 \mathrm{~dB}$, and thus we can estimate that this difference includes errors from the noncalibrated probe and mismatched instrument. Furthermore, (4) utilized for EIRP calculation has weakness as similar to the absolute measurement method among the gain measurement method [20], which means that the error of each factor of (4) directly affects the value of EIRP.

For another example, the base-station antenna operating at $859 \mathrm{MHz}$ was experimented in a small anechoic chamber as shown in Figure 8(a). The antenna dimensions were $0.3 \mathrm{~m}$ $\times 0.7 \mathrm{~m}$ in the $x$ - and $y$-directions, respectively. The farfield gain of the base-station antenna was $G_{t}=12.38 \mathrm{dBi}$, and the input power to the base-station antenna was $P_{t}=$ $-1.08 \mathrm{dBm}$. Therefore, the exact EIRP was $11.30 \mathrm{dBm}$. The probe gain was $G_{\text {probe }}=4.20 \mathrm{dBi}$ at $859 \mathrm{MHz}$. The separation distance between the base-station antenna and probe is $R_{1}$ $=0.6 \mathrm{~m}$. The phase-retrieval technique and Fresnel-to-Far transformation were performed, and, finally, the transformed far-field pattern is drawn in Figure 8(b) with the reference farfield pattern. The normalized patterns agreed closely over the valid range of $\theta=-30^{\circ}$ to $+30^{\circ}$. The shortage of scanning data also brings the disagreement out of the valid angle. The transformed $P_{R_{2}}$ at $\theta=0^{\circ}$ was $-33.07 \mathrm{~dB}$ at $R_{2}=7.0 \mathrm{~m}$, and thus EIRP $=10.75 \mathrm{dBm}$ from (4). The difference between the transformed and reference EIRPs was $0.55 \mathrm{~dB}$, which proves again the validity of the proposed method in this paper.

\section{Conclusion}

We proposed a practical EIRP measurement technique for a base-station antenna installed in a real environment. A Fresnel region measurement method was chosen to remove multipath interference. A simple phase-retrieval technique provided with priori information is proposed, and thus the data acquisition is required along only a single line without moving a measurement vehicle. For verification of the proposed method, a computer simulation and experiment in a small anechoic chamber have been performed. The transformed far-field pattern and EIRP agreed closely with those of the reference data within a valid angle. Currently, an outdoor measurement system for a base station is being built, and the proposed method in this paper will be adopted. The research on the effect of the probe directivity and the distance between the probe and base-station antenna is also ongoing.

\section{References}

[1] "IEEE recommended practice for local and metropolitan area networks. Coexistence of fixed broadband wireless access systems," IEEE Standard 802.16.2-2004, 2004.

[2] "Basic standard for the calculation and measurement of electromagnetic field strength and SAR related to human exposure from radio base stations and fixed terminal stations for wireless telecommunications system $(110 \mathrm{MHz}-40 \mathrm{GHz})$, , BS EN 50383:2002, 2002.

[3] "Measurement methods of exposure to base stations," TTAS .KO-06.0125, 2006.

[4] "Electromagnetic compatibility and Radio spectrum Matters (ERM); wideband transmission systems; data transmission equipment operating in the $2,4 \mathrm{GHz}$ ISM band and using wide band modulation techniques; harmonized EN covering the essential requirements of article 3.2 of the R\&TTE directive," ETSI EN 300 328, v1.8.1, 2012.

[5] “Off-axis EIRP envelopes for FSS earth station operations," FCC CFR title 47: 25.218, 2009.

[6] D. M. Pozar, Microwave and RF Design of Wireless Systems, John Wiley \& Sons, New York, NY, USA, 2000.

[7] A. Catalani, F. D. Paolo, M. Migliorelli, L. Russo, G. Toso, and P. Angeletti, "Ku band hemispherical fully electronic antenna for aircraft in flight entertainment," International Journal of Antennas and Propagation, vol. 2009, Article ID 230650, 7 pages, 2009.

[8] R. V. Gatti, L. Marcaccioli, E. Sbarra, and R. Sorrentino, "Flat array antennas for Ku-band mobile satellite terminals," International Journal of Antennas and Propagation, vol. 2009, Article ID 836074, 5 pages, 2009. 
[9] B.-J. Jang, S. Moon, and J.-B. Lim, "EIRP measurements of WCDMA base station using pilot channel in line-of-sight environments," Journal of the Institute of Electronics Engineers of Korea, vol. 22, no. 5, pp. 545-551, 2011.

[10] S. Gregson, J. McCormick, and C. Parini, Principles of Planar Near-Field Antenna Measurements, IET, Herts, 2007.

[11] O. M. Bucci, G. D’Elia, G. Leone, and R. Pierri, "Far-field pattern determination from the near-field amplitude on two surfaces," IEEE Transactions on Antennas and Propagation, vol. 38, no. 11, pp. 1772-1779, 1990.

[12] G. D’Elia, R. Pierri, and F. Soldovieri, “A Novel approach to nearfield far-field transformation from intensity data," in Proceedings of the 1998 IEEE International Antennas and Propagation Symposium and USNC/URSI National Radio Science Meeting. Part 1 (of 4), pp. 1335-1338, June 1998.

[13] S. F. Razavi and Y. Rahmat-Samii, "On the uniqueness of planar near-field phaseless antenna measurements based on two amplitude-only measurements," in Proceedings of the IEEE International Symposium on Antennas and Propagation and USNC/URSI National Radio Science Meeting (APSURSI '08), July 2008.

[14] G. D'elia, G. Leone, R. Pierri, and G. Schirinzi, "New method of far field reconstruction from Fresnel field," Electronics Letters, vol. 20, no. 8, pp. 342-343, 1984.

[15] S. S. Oh, J. M. Kim, and J. Yun, "Antenna measurement on cylindrical surface in fresnel region using direct far-field measurement system," ETRI Journal, vol. 29, no. 2, pp. 135-141, 2007.

[16] S. S. Oh and J. I. Moon, "Measurement guideline of Fresnel-field antenna measurement method," ETRI Journal, vol. 31, no. 1, pp. 68-70, 2009.

[17] S. S. Oh and J. H. Yun, "New method for predicting the electromagnetic field at a finite distance using fresnel field transformation," IEEE Antennas and Wireless Propagation Letters, vol. 7, pp. 291-293, 2008.

[18] "High frequency structure simulator, version 12," Ansoft HFSS, 2009.

[19] F. J. Cano-Facila, S. Pivnenko, and M. Sierra-Castaner, "Reduction of truncation errors in planar, cylindrical, and partial spherical near-field antenna," International Journal of Antennas and Propagation, vol. 2012, Article ID 438727, 19 pages, 2012.

[20] J. D. Kraus and R. J. Marhefka, Antennas for All Applications, McGraw-Hill, Boston, Mass, USA, 2002. 

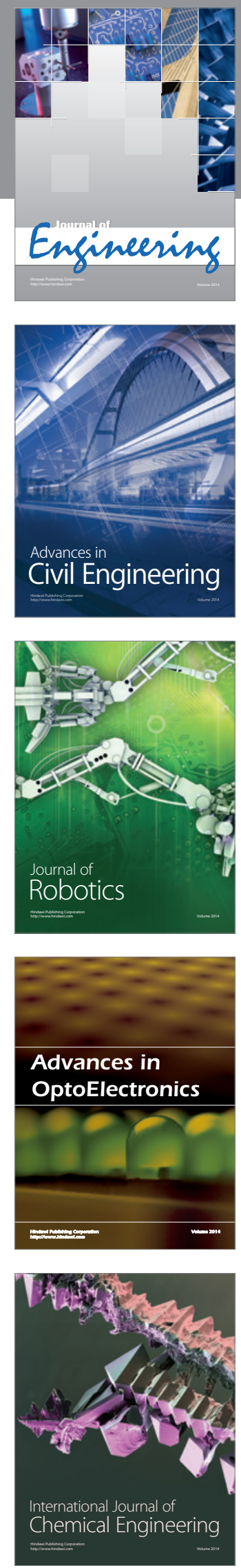

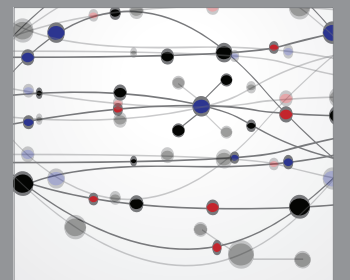

The Scientific World Journal
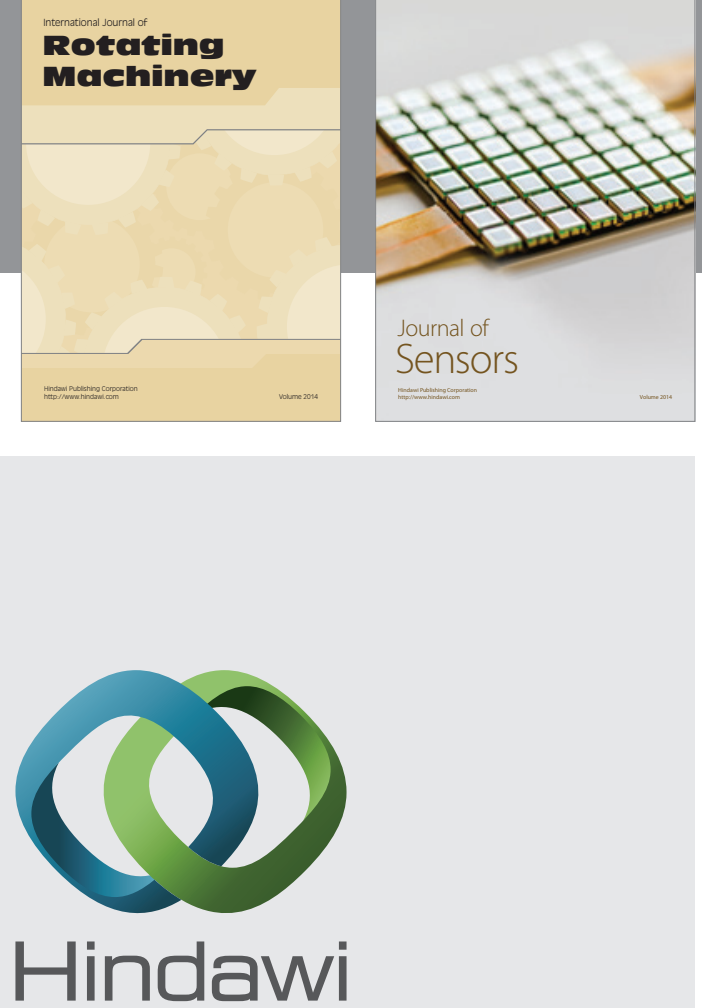

Submit your manuscripts at http://www.hindawi.com
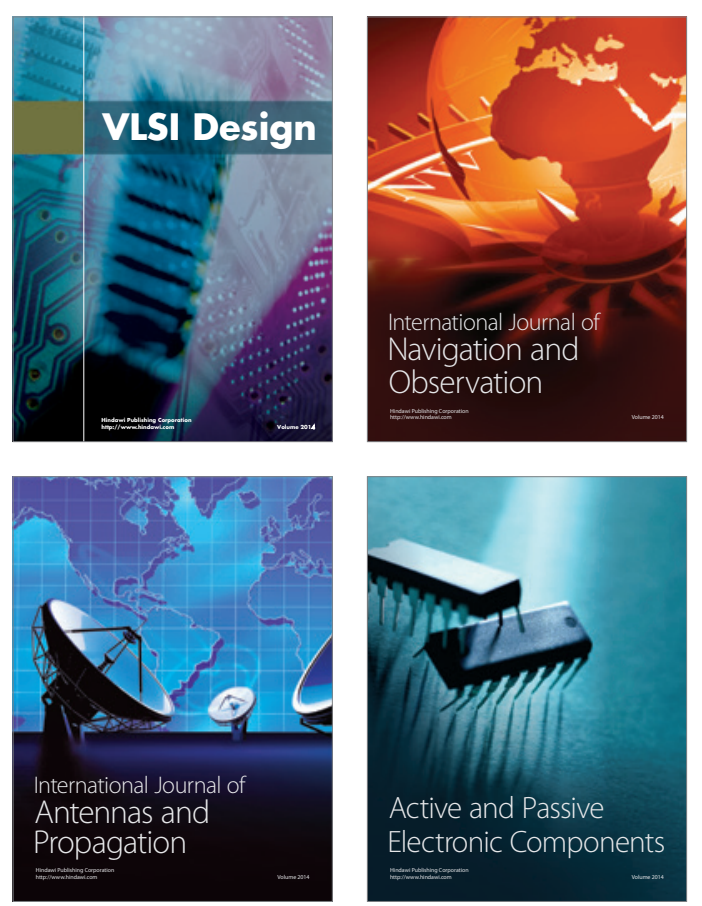
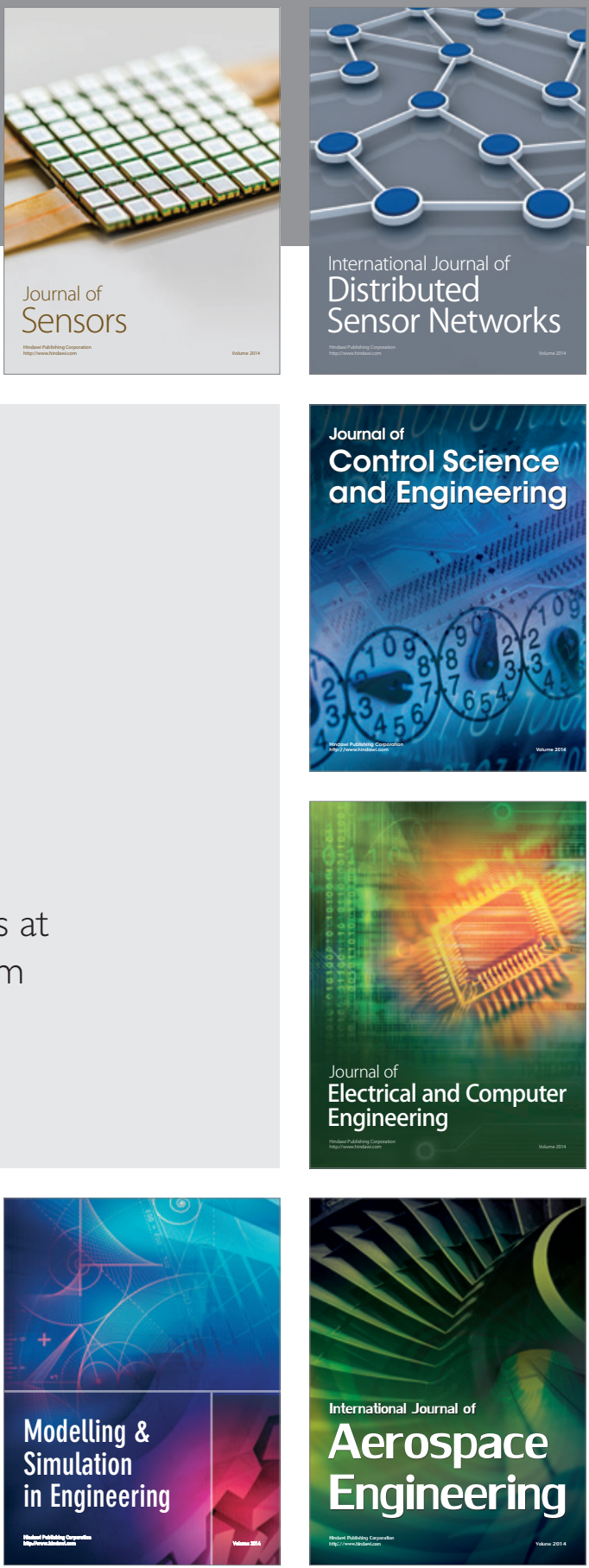

Journal of

Control Science

and Engineering
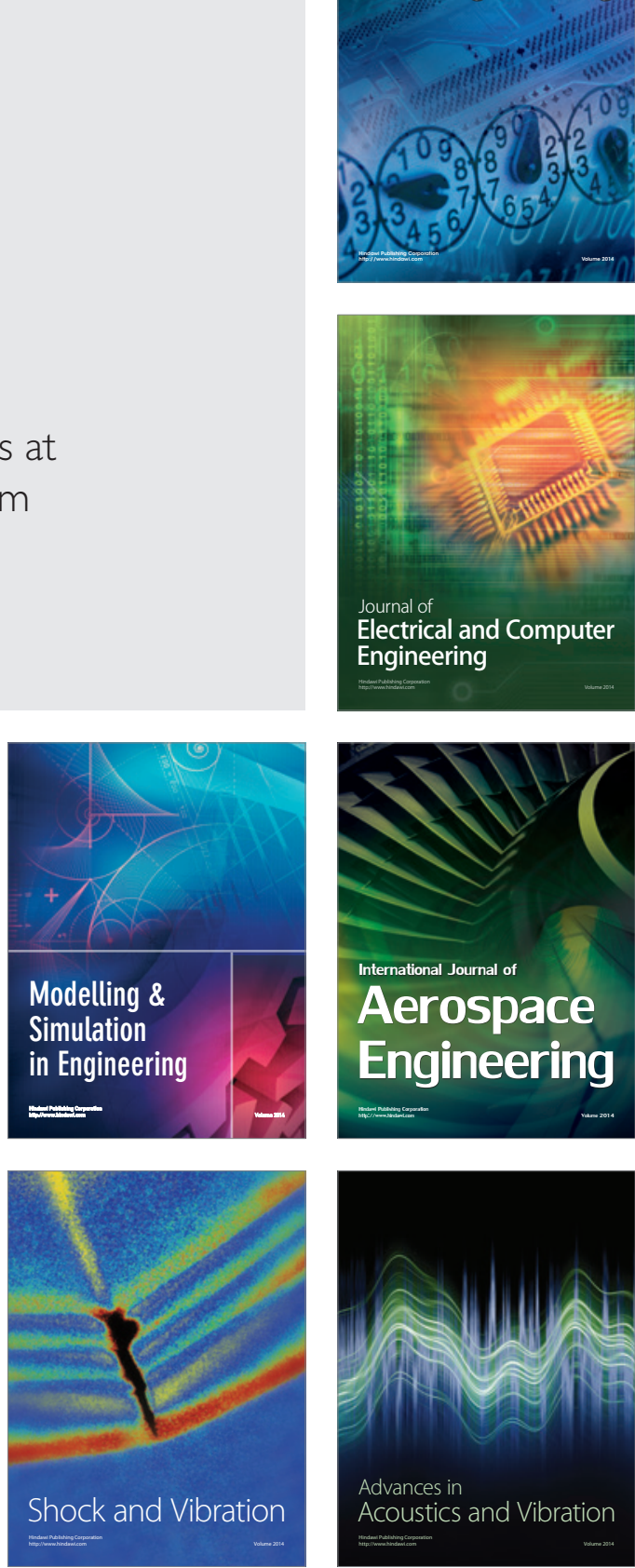